\title{
Practitioner based quality improvement: a review of the Royal College of Nursing's dynamic standard setting system
}

\author{
Clare Morrell, Gillian Harvey, Alison Kitson
}

\begin{abstract}
Objective-To explore and describe the implementation of the Royal College of Nursing's approach to audit-the dynamic standard setting system-within the current context of health care, in particular to focus on how the system has developed since its inception in the 1980s as a method for uniprofessional and multiprofessional audit.
\end{abstract}

Design-Qualitative design with semistructured interviews and field visits.

Setting-28 sites throughout the United Kingdom that use the dynamic standard setting system.

Subjects-Quality and audit coordinators with a responsibility for implementing the system; clinical staff who practice the system.

Main measures-Experiences of the dynamic standard setting system, including reasons for selection, methods of implementation, and observed outcomes. Results-Issues relating to four themes emerged from the data: practical experiences of the system as a method for improving patient care; issues of facilitation and training; strategic issues of implementation; and the use of the system as a method for multiprofessional audit. The development of clinical practice was described as a major benefit of the system and evidence of improved patient care was apparent. However, difficulties were experienced in motivating staff and finding time for audit, which in part related to the current format of the system and the level of training and support available for clinical staff. Diverse experiences were reported in the extent to which the system had been integrated at a strategic level of quality improvement and its successful application to multiprofessional clinical audit.

Conclusions-The Royal College of Nursing's dynamic standard setting system can successfully be used as a method for clinical audit at both a uniprofessional and multiprofessional level. However, to capitalise on the strengths of the system, several issues need to be considered further. These include modifications to the system itself, as well as a more strategic focus on resources and support for audit, better integration of quality initiatives in health care, and a continuing focus on ways to achieve true multiprofessional collaboration and involvement of patients in clinical audit. (Quality in Health Care 1997;6:29-34)

Keywords: standard setting; clinical audit; implementation.

\section{Introduction}

BACKGROUND

Along with many other professional bodies the Royal College of Nursing has been involved in work on standards of health care for many years. This began with a major research project in the 1960s, known as the Study of Nursing Care, ${ }^{1}$ which was set up to define standards for nursing care. This was followed by the establishment of a Standards of Care Working Committee. $^{23} \mathrm{~A}$ recommendation of this committee led to the setting up of a specific programme of work in 1985 called the Standards of Care Project (now known as the Dynamic Quality Improvement Programme). The initial remit of this project was to develop a practical framework and system which clinical staff could use to evaluate their own standards of care.

The system that emerged was an approach to audit based on criteria, known as the "dynamic standard setting system" (sometimes called DySSSy). This system allows nurses and other healthcare professionals to set and audit their own standards. It developed from the integration of several concepts of healthcare quality including Donabedian's description of structure, process, and outcome, ${ }^{4}$ the notion of a quality assurance cycle, ${ }^{5}$ and the work of the Manitoba Association of Registered Nurses ${ }^{6}$ in developing standards based on criteria. In the United Kingdom, the work was developed and tested in practice, ${ }^{7}$ and then translated into a framework, ${ }^{8}$ which was subsequently published as a set of teaching manuals in $1990 .^{\circ}$

The dynamic standard setting system provides an explicit set of principles for developing patient care. The system states that quality improvement should be focused on the patient, be led by those who give care, and should take place at the closest point to service delivery. The system promotes improvements by solving specific problems. Standards are defined for specific clinical situations and written as achievable levels of care. As the focus is on patient care the importance of multiprofessional collaboration is also emphasised. ${ }^{8}$ 
Implementing the dynamic standard setting system at a practical level is based on a continual cycle of defining, measuring, and taking action. Similar versions of this cycle are variously described as the quality assurance cycle,${ }^{5}$ the quality improvement cycle, ${ }^{10}$ the medical audit cycle, ${ }^{11}$ the nursing audit cycle, ${ }^{12}$ and the clinical audit cycle. ${ }^{13}$

In the defining phase of the system a group of practitioners come together to form a quality improvement team which works to improve specific topics. The composition of the group should reflect the topic under study and be representative of all those involved in delivery of the service.

The dynamic standard setting system is set out with clear guidance for its implementation. Local groups should be supported by a facilitator when considering the stages of the quality improvement cycle. The main role of a facilitator is to support the group processes. The facilitator is explicitly trained to have knowledge and skills in group dynamics, group processes, and consensus decision making.

Within the overall framework of the system, facilitators are identified at three levels: local facilitators who work with clinical teams to support the implementation of standards and audit in practice; key facilitators who oversee and coordinate the quality programme, often taking on a specific responsibility for education, training, and information; and expert facilitators who introduce and plan strategic quality improvements.

Local facilitators are often clinical staff who undergo training to enable them to take on that role within a quality improvement team. Key and expert facilitators are likely to be in posts in which there is an explicit responsibility for quality improvement and audit.

Once a group is formed to work on a topic forms are used for each stage of the cycle. The defining phase of quality improvement involves the development of a statement on standards which reflects the overall objective of the exercise. From this statement, criteria necessary to implement the standard are identified. These are the resources required, the activities undertaken by staff, and the anticipated results of an intervention for the patients. The criteria are classified and recorded according to the Donabedian framework of structure, process, and outcome criteria. ${ }^{4}$

To measure and compare practice with the standard, the second phase of the cycle involves the development of an audit form to monitor the achievement of the structure, process, and outcome criteria. A sample is identified, together with data collection methods, a time frame for the collection of data, and the people responsible. Implementation and audit dates for the standard and criteria are then negotiated by the group in consultation with the wider healthcare team.

The final phase of the cycle involves action planning. Data are summarised and brought back to the group for them to interpret the findings and decide on what action (if any) is needed. Actions are prioritised and people are given responsibility for seeing that plans are carried out in an agreed period and a date for reaudit is negotiated.

DYNAMIC STANDARD SETTING SYSTEM IN RELATION TO OTHER INITIATIVES

The dynamic standard setting system shares many common characteristics with other methods of clinical audit and quality improvement. Its distinguishing features can be summarised as follows:

(1) It is led clinically as opposed to managerially, although it is essential that it is supported by the organisation.

(2) It is locally based, emphasising the full participation of nurses and other healthcare professionals in all three phases of the cycle.

(3) It uses small group processes within the local quality improvement team to ensure commitment to developing practice.

(4) There is a clearly identified role for the facilitator, guiding and supporting the local group process; the facilitator is a skilled member of the team who is specifically trained in promoting effective group work.

(5) It generates explicit standards and criteria to implement elements of structure, process, and outcome for each audit topic.

\section{UPTAKE OF THE DYNAMIC STANDARD SETTING} SYSTEM

With its origins in the mid-1980s, the system was first introduced to nurses before healthcare audits became compulsory. It was used in many specialty areas and in diverse topics - for example, care of paediatric patients before an anaesthetic, ${ }^{14}$ incidence of pressure sores in elderly people, ${ }^{15}$ and maintenance of privacy in care of those with a learning disability. ${ }^{16}$

The rapid uptake and enthusiasm among nurses in the United Kingdom led to the development of the quality assurance network (now known as the DQI network). This was established to provide its members with information, training, and support as they implemented the system in their areas. The network has spread into Europe (Euroquan) and both networks continue to provide healthcare staff with a forum for exchange of information on a wide variety of issues on quality.

The dynamic standard setting system proved to be both popular and effective among clinical staff and was commended by independent studies as a valuable method for quality improvement based on practice. ${ }^{17}{ }^{18}$ At the same time, critics of the approach questioned the basic premise of allowing practitioners to set and audit their own standards of care and expressed concern over the reliability and validity of criteria derived in this way. ${ }^{19}$ Others viewed the system as being potentially wasteful of time and labour, due to its local, as opposed to national, focus. ${ }^{20}$ These concerns were considered further in an experimental study undertaken between 1989 and 1992 to 
evaluate the impact of the dynamic standard setting system on clinical practice and patients in an acute surgical setting. ${ }^{21}$

AIMS OF THE STUDY

As the impact and effect of the reforms on health care became evident a growing need was recognised to evaluate how the system had been incorporated into quality strategies, contract monitoring, the patient's charter, and clinical audit. This was especially important given the differences in terminology that were introduced. Nursing had traditionally used the language of quality assurance and setting standards, as opposed to audit. Although semantically, in the way they were being applied, there was little or no difference between the terms, it was of interest to assess whether the system was perceived as one which could support audit in practice, particularly with the growing focus on multiprofessional clinical audit.

The dynamic standard setting system has been widely implemented both in the United Kingdom and abroad. There are many cases that describe how the system has enabled nurses to improve patient care. In an article describing how a team of clinical staff improved rest and relaxation for patients in intensive care, Dunn (1990) ${ }^{22}$ wrote:

"Standards of care have enabled us to communicate our expectations to others. We have been able to identify deficiencies in resources and hold on to those necessary to maintain the quality of care. By sharing ideas and tackling problems in a co-ordinated way we have made progress towards improving practice and clarifying our nursing care priorities alongside the demands which are made by high-tech medicine."

Our paper outlines an evaluation study conducted during 1994 to investigate the implementation of the Royal College of Nursing's approach to audit-the dynamic standard setting system and discusses ways in which it might be developed for future use in multiprofessional clinical audit and clinical effectiveness.

\section{Methods}

An evaluation study was set up in 1994 to explore and describe the development and implementation of the dynamic standard setting system within present day health care. Semistructured interviews were undertaken with a sample of nurses involved in implementing the system across a trust or provider unit.

Interviews covered issues of implementation of the system-such as the reasons for its selection, how the system fitted with other initiatives within the organisation, what training was available for staff, how standards were validated, how results were reported, how success was defined, and which staff were involved.
SELECTING A SAMPLE

The interview sample was drawn from the Royal College of Nursing Dynamic Quality Improvement network which has a membership of over 3000 throughout the United Kingdom. The sample comprised nursing staff with a specific responsibility for coordinating quality and audit programmes led by nurses and other healthcare professionals. Most of the sample were senior nurses in posts responsible for nursing quality. Some were nursing managers with operational responsibility, and others were employed with a wider remit for quality within their organisation. These people could be seen to be key facilitators in organising the implementation of the system, by both promoting and supporting it.

Visits were made to each organisation to hear the views of staff actively involved in local groups that used the system. The emphasis was placed on the interviews with the key facilitators as they were considered to have the best overview of the successes and problems within the organisation. Selection aimed to be representative of regions across the United Kingdom, to reflect a variety of experience in use of the system, and to include those who had found difficulties with it. Twenty eight trusts and provider units were visited and interviews with key facilitators were recorded on tape and transcribed. Field notes were used to record information from conversations with clinical staff.

\section{DATA ANALYSIS}

Data were analysed with a form of content analysis. This coding process was repeated on a $50 \%$ sample of the interview transcripts by the project supervisor to maximise reliability between raters.

\section{Results}

The findings have been divided into four themes, which emerged from the data. These were practical experiences of the system as it was used to evaluate and improve patient care, facilitation and training used in the system, strategies of implementation, and the system as a method of multiprofessional audit.

PRACTICAL EXPERIENCES OF THE DYNAMIC STANDARD SETTING SYSTEM AS A METHOD FOR IMPROVING PATIENT CARE

A range of benefits and difficulties of the system were identified. Many of the ideas expressed by staff were contradictory and seemed to be linked to organisational, philosophical, and cultural issues.

The dynamic standard setting system was most commonly selected because of the principles on which it is based-namely, a system designed for clinical staff; that involved nurses and other healthcare professionals; resulted in continuous improvement; and could solve local problems.

Several key facilitators had selected the dynamic standard setting system because it was thought to be suited to multiprofessional work. However, this seemed to challenge the views of another group, who had selected the system 
because it was written by nurses for nurses and was seen to be allied to the Royal College of Nursing.

The development of clinical practice was described as the overriding benefit of involvement with the system. Evidence from successive audits showed progress in clinical care in many areas. An example of this is shown in the box.

The dynamic standard setting system was also used for professional development of staff. Reported benefits included self criticism of actions, an increased ability to take constructive criticism from peers, and a growing awareness of the wider issues of quality within organisations.

The emphasis on group work was also seen to have advantages. Teams of multiprofessional staff began to define goals and work together on projects; in the words of one interviewee

\section{"Instead of ranting and raving at each other down the phone you can come together and talk."}

Staff had gained an appreciation of each others' roles and their confidence to participate in multiprofessional groups had increased.

Several interviewees perceived the dynamic standard setting system as adaptable and had changed the system to meet their needs. Two of the forms were commonly omitted, with practitioners moving straight from a combined audit form and audit record and then to an action plan that included the audit summary.

Finding sufficient time in a hectic clinical schedule was universally reported as a major problem. The experience of those questioned suggested that the initial implementation of the system was very time consuming, but once staff had developed skills in its use the process could be streamlined and incorporated into regular activities.

This lack of time seems to be common to all initiatives to improve practices in the current climate, but specific complaints were made about the amount of paperwork involved in the system.

The other main difficulty experienced was the attitude of staff; many remaining unconvinced that participation would be beneficial and perceiving the system as an inspection or a counting exercise. Interviewees reported spending much time trying to encourage staff to become involved.

Involvement of patients in the system was sporadic. Most of the staff interviewed talked

In one site the nursing team had become concerned about the duration of preoperative fasting. After reviewing publications and consulting with anaesthetists and surgeons, practice was revised to meet an agreed standard and criteria were set. Successive audits showed considerably fewer patients being starved beyond the recommended period. about their standards being patient centred and described encouraging patients to express their opinions as a part of the monitoring of standards. Rarely were patients actually involved as team members in the entire process. This was reported to be changing, with plans for their participation in focus groups and topic suggestion boxes in several organisations.

From the sample interviewed, mental health seemed to have led the way in involving patients in the system. One mental health trust visited had an active group of patients who were participating in the system. They were paid a set fee plus travelling expenses to attend ward standard setting meetings and represent patients' views. One such patient described with enthusiasm his experiences of being involved in standard setting. He thought that his input had been valued and had appreciated the opportunity to learn more about the issues faced by healthcare staff.

ISSUES OF FACILITATION AND TRAINING

From the interviews it seemed clear that the personality, skills, and attributes of the person operating as the key facilitator are highly influential in the implementation and success of the system. The willingness of clinical staff to become involved in quality improvement often seemed to be influenced by whether the key facilitator was liked and respected. Also, for that person to function effectively, organisational support from managers was thought to be crucial.

Interviewees enjoyed being facilitators and they were glad to be involved in development of practice. Several also described how they had developed personally and professionally through the role. Others felt overwhelmed and isolated, struggling to meet the demands placed on them both by senior management and clinical staff.

To introduce the dynamic standard setting system across an organisation a system of local facilitators, specifically trained and supported by local management in that role, seemed to provide a successful model, allowing the key facilitator to function as mentor and coordinator. Staff nurses and other non-medical members of the professions allied to medicine were the most usual local facilitators, but patients, ancillary staff, and students had also been trained for the role. None of those interviewed reported training medical staff as facilitators.

The qualities of the most successful facilitators seemed to be good communication skills, an ability to empower others, and a willingness to challenge the status quo. Motivation, enthusiasm, and commitment to developing patient care were described as more important criteria for selection of local facilitators than an in depth knowledge of the system.

More training and education was an almost universal request, both for skills needed in group work and for facilitation - the main barriers being funding for the training itself and releasing staff from clinical duties. The 
importance of integrating quality and audit into basic and subsequent education was also highlighted.

STRATEGIC ISSUES OF IMPLEMENTATION

The dynamic standard setting system was considered to form a useful part of a trust's strategy on quality, but could not stand alone. Interviewees fell into three groups.

(1) Those for whom the system had become integral to their trust's strategy on quality.

(2) Those who had linked the system to local practice development projects, without reference to the trust's strategy.

(3) Others for whom the system seemed to be running alongside other quality initiatives, with no clear links.

Those who had been using the system before the introduction of the health service reforms considered themselves in a strong position to develop a quality strategy based on the progress achieved by the system approach. One trust that had a well established system integrated it by basing the quality specifications for the contracts on its standards.

However, a fragmented approach to quality strategy usually persisted. Examples were cited of many quality activities being introduced piecemeal. Commonly, one initiative would be funded by the trust executive board, another from regional audit money, and another from the nursing budget, and no one had taken the responsibility for bringing these various initiatives together within an integrated strategic plan. Such an integrated approach to quality seemed to require not only clear leadership but also the full commitment of the management team in establishing a set of systems and structures to implement the different initiatives.

THE SYSTEM AS A METHOD FOR MULTIPROFESSIONAL AUDIT

In many of the interviews there were misunderstandings and boundaries between the different professions, which presented serious obstacles to the development of collaborative audit. Many of the problems revolved around issues of methodology and were seen to relate to the historical development of approaches to audit of the different professions. Joint educational initiatives were found to be a good way of overcoming such barriers, with training for quality improvement and audit focused at clinical teams.

Involvement of medical staff in the system most commonly consisted of their comments on draft documents as they seemed to be reluctant to join groups. Interviewees complained that medical staff had problems in being members as opposed the leaders of teams and they found it hard to sit and listen to all points of view. Lack of time was again raised as a main barrier to effective communication between professional groups.

Despite the various problems encountered, there were examples of teams successfully using the system as a model for multiprofessional audit. These included:
- A multiprofessional approach to care of diabetic patients

- Social workers and community psychiatric nurses developing systems for clinical supervision

- Multiprofessional standards on care of pressure areas

- Occupational therapists, speech therapists, dieticians, and nurses developing nutritional care

- Pharmacists, doctors, and nurses auditing the use of night sedation.

It is important to note that successful multiprofessional audit projects that used the system could fail to be reported, for example, to purchasers, due to a lack of an integrated strategy on quality or good communication channels.

\section{Discussion and recommendations}

Ten years since its development, the dynamic standard setting system continues to be used to provide an effective method for promoting improvements in patient care and can be a useful way to audit at both a uniprofessional and multiprofessional level.

A wide spread of issues on the implementation and integration of the system has emerged. These indicate the need for discussion at different levels to consider a broad range of national and local issues.

At a clinical level, the commonly expressed difficulty of finding time for involvement in audit should be rectified. With the dynamic standard setting system, this could be initially considered by streamlining the system to reduce the amount of paperwork involved. This would enable those staff with a good understanding of the whole process to minimise the steps taken for each audit project.

Time could also be saved by using a software programme-for example, DQI Toolbo-to accompany the system. Time for involvement in clinical audit activity is a fundamental problem within present day health care and one highlighted by other evaluation studies. For example, a study undertaken jointly by Brunel University and King's College, London ${ }^{23}$ identified a lack of time as a barrier to the development of clinical audit as perceived by staff in the professions allied to medicine. This suggests a need to consider time at a strategic level of healthcare planning, as well as at the clinical level.

Further work is also required to promote a positive attitude to audit and quality improvement. This is an area where people functioning as key facilitators have a potentially important contribution to make, providing training, raising awareness, and motivating staff to become involved in audit. It is essential that those people selected to function in this role themselves possess the necessary skills and knowledge to encourage and support others.

In general more training on audit and quality improvement is needed. The provision of more team based training could help enhance developments in multiprofessional audit. Involvement of patients in audit should also be developed. To consider the range of issues relating 
to training, multiprofessional collaboration, and patient participation, the Royal College of Nursing could usefully continue to develop its links with other professional organisations and patients' organisations to explore joint educational initiatives.

At a strategic level, there is a clear need for better integration of the various quality improvement and audit initiatives that take place alongside each other. This is particularly important in the light of initiatives being introduced as a result of the emphasis on clinical effectiveness and evidence based health care. This requires a better understanding and overview of the range of quality initiatives taking place within a trust to ensure that they make the most effective contribution to the overall organisational strategy for promoting quality patient care.

This is an area also highlighted by the CASPE report on the implementation of medical audit in provider units. The study reported that

"In most provider units clinical audit has grown alongside other important and long established quality programmes. At present, few providers have succeeded in linking these different and disparate initiatives."24

It would seem, therefore, that structures and systems need to be introduced to ensure better integration of activities and initiatives related to quality.

\section{Conclusion}

The dynamic standard setting system, as in other approaches to quality improvement and audit, has the potential to considerably improve patient care and provides a framework that can be used by patients, nurses, and other professional groups alike. Some modifications could be made to the system to improve its usefulness. However, for success, as illustrated by this and previous research studies, ${ }^{21} 25$ it must be implemented in an organisation in which clear structures and systems exist to promote and support quality improvement initiatives at both the clinical and strategic levels of health care.

With the advent of league tables and the continuing demand for information from central government there is a risk that the place for internal approaches to quality improvement could become eroded in favour of external inspection. Both in the United States and in the United Kingdom the uptake of locally based methods came in response to practitioners' objections to external inspection. Unless work is done to encourage, value, and integrate internal quality projects within organisations we could see the pendulum swing all the way back again

We thank the staff of the clinical sites that participated in the study. Our thanks are also extended to those who were involved in the pilot stages of the research. The full version of this report can be found in Morrell C, Harvey G, Kitson A. The reality of practitioner-based quality improvement. Report No 14. Oxford: National Institute for Nursing, 1995.

1 McFarlane J. The proper study of the nurse. London. Royal College of Nursing, 1970

2 Royal College of Nursing. Standards of nursing care. London Royal College of Nursing, 1980.

3 Royal College of Nursing. Towards standards. London: Roya College of Nursing, 1981.

4 Donabedian A. Evaluating the quality of medical care. Mil bank Memorial Fund Quarterly 1966;44:166-206

5 Lang NM. Quality assurance in nursing. AORN fournal 1975;22:180-6.

Scherer K. Satisfaction guaranteed. Nursing Times 1985; 81:32-3.

Kendall H, Kitson A L. Quality assurance - rest assured. Nursing Times 1986;82:29-31.

8 Kitson AL. A frameworkfor quality: a patient-centred approach to quality assurance in health care. Harrow: Scutari, 1989.

9 Royal College of Nursing. Quality patient care: the dynamic Royal College of Nursing. Quality patient care:
standard setting system. Harrow: Scutari, 1990.

10 Royal College of Nursing. DySSSy tutorial. Oxford: RCN Dynamic Quality Improvement Programme, 1994

11 Shaw C. Medical audit: a hospital handbook. London: King' Fund Centre, 1991.

12 Department of Health. Measuring the quality - nursing car audit teaching notes. London: DoH, 1990.

13 Department of Health. Clinical audit: meeting and improving standards in health care. London: $\mathrm{DoH}, 1993$.

14 Girvin J. Standard setting in paediatrics. Nursing 1990 $4: 26,28$.

15 Girvin J. Using DySSSy - a case study. In: Royal College of Nursing. Quality patient care: the dynamic standard setting Nursing. Quality patient care: the dynamic standard setting

16 Marr $H$, Pirie M. Protecting privacy. Nursing Times 990;86:58-9.

17 Audit Commission. The virtue of patients: making best use of ward nursing resources. London: HMSO, 1991.

18 Audit Commission. Making time for patients: a handbook for ward nursing sisters. London: HMSO, 1992

19 Goldstone LA. A very peculiar practice. Nursing Times 1991;87:41-3.

$20 \mathrm{Kemp}$ N, Richardson E. Quality assurance in nursing practice. Oxford: Butterworth-Heinemann, 1990.

21 Kitson A, Harvey G, Hyndman S, Sindhu F, Yerrell P. The impact of a nursing quality assurance approach, the dynamic standard setting system (DySSSy) on nursing practice and patient outcomes (the ODySSSy project). Report No 4 Oxford: National Institute for Nursing, 1994 .

22 Dunn C. Improving intensive care. Nursing Times 1990, 86:32-4.

23 Kogan M, Redfern S. Making use of clinical audit. Buckingham: Open University Press, 1995.

24 Buttery Y, Walshe K, Coles J, Bennett J. The development of audit: findings of a national survey of health care provider unit in England. London: CASPE Research, 1994.

25 Harvey G, Kitson A. Achieving improvement through quality: an evaluation of key factors in the implementation process. F Adv Nurs 1996;24:185-95. 